\title{
ON THE CLOSURE OF THE DISCRETE SPECTRUM OF NUCLEARLY PERTURBED OPERATORS
}

\author{
FRANZ HANAUSKA
}

Abstract. Let $Z_{0}$ be a bounded operator in a Banach space $X$ with purely essential spectrum and $K$ a nuclear operator in $X$. Using methods of complex analysis we study the set of accumulation points of the discrete spectrum of the operator $Z:=Z_{0}+K$. We formulate conditions for $Z$ to exclude certain points or subsetes of the essential spectrum of $Z$ to be accumulation points of the discrete spectrum. These results are applied to the operator of multiplication perturbed by integral operators with continuous kernel and to the discrete Laplacian perturbed by nuclear Jacobi operators.

Mathematics subject classification (2010): 26D15, 26A51, 32F99, 41A17.

Keywords and phrases: Eigenvalues, discrete spectrum, nuclear perturbations.

\section{REFERENCES}

[1] E. B. Davies, Linear operators and their spectra, Cambridge University Press, Cambridge 2008.

[2] M. Demuth \& F. HANAuSKa, On the distribution of the discrete spectrum of nuclearly perturbed operators in Banach spaces, to be published in Indian J. Pure Appl. Math.

[3] I. C. Gohberg, S. Goldberg \& M. A. KAAShoeK, Classes of linear operators, Vol. 1. Birkhäuser Verlag, Basel 1990.

[4] I. C. Gohberg, S. Goldberg \& N. Krupnik, Traces and determinants of linear operators, Birkhäuser Verlag, Basel 2000.

[5] T. Kato, Perturbation theory for linear operators, Springer-Verlag, Berlin 1995.

[6] R. Remmert \& G. Schumacher, Funktionentheorie I, Springer, Berlin - Heidelberg, 6. edition, 2002. 